\title{
Metachronous Primary Cerebral Rhabdomyosarcoma After Treatment of Oral Primitive Neuroectodermal Tumor/ Ewing's Sarcoma in a School-Aged Girl: A Case Report
}

Danielle Anne Gonong and Manuelito Madrid

Philippine Children's Medical Center, Quezon City, Philippines

\section{ABSTRACT}

Multiple primary malignancies are defined as two or three malignant neoplasms arising in different organ systems. Several cases of multiple primary malignancies are emerging in recent years due to the advancement in medical therapy and diagnostics. Multiple primary malignancies are not uncommon occurring at $0.7-16 \%$ of cancer patients, however reported cases of multiple primary sarcomas are sparse. Presented in this report is a pediatric patient diagnosed with primary metachronous cerebral rhabdomyosarcoma after being treated for primitive neuroectodermal tumor/Ewing's sarcoma of the oral cavity. Despite limited cases addressing multiple primary sarcomas, this entity must not be overlooked as it is associated with a meager outcome compared to an index case of sarcoma alone.

Key words: multiple primary malignancies, multiple primary sarcomas, metachronous malignancies, PNET/ Ewing's sarcoma, rhabdomyosarcoma, Immunohistochemistry, fluorescence in situ hybridization, reverse transcription-polymerase chain reaction

ISSN 2507-8364 (Online)

Printed in the Philippines.

Copyright $\odot 2020$ by the PJP.

Received: 30 November 2020.

Accepted: 26 December 2020.

Published online first: 29 December 2020.

https://doi.org/10.21141/PJP.2020.13

Corresponding author: Danielle Anne G. Gonong, MD

E-mail: danielle.anne.gonong@gmail.com

\section{INTRODUCTION}

Multiple primary malignancies (MPM) are an emerging clinical entity. ${ }^{1}$ These are further divided into synchronous and metachronous malignancies, temporally separated by six months. ${ }^{2-5}$ Presently, there are adequate number of scholarly articles on MPM, however reported cases of multiple sarcomas are rare. This is especially uncommon in the pediatric population, and based on the authors literature search, there are no other reported case of multiple primary sarcomas in the head and neck region and central nervous system occurring in the pediatric population.

\section{CASE REPORT}

This is a case of an 11-year-old girl diagnosed with primitive neuroectodermal tumor (PNET)/Ewing's sarcoma stage IV of the left oral cavity mass on September 2017. She has no family history of malignancy nor known environmental exposure to carcinogens. On December 2018, the patient was in remission after undergoing 7 cycles of PNET/ Ewing's sarcoma chemotherapy protocol and 30 cycles of radiation therapy (RT). Monitoring cranial and chest computerized tomography (CT) scan done on January 25, 2019 showed, normal study of the brain, polysinusitis, soft tissue fullness on the left pterygoid muscles and left parapharyngeal space. The chest CT scan shows unremarkable findings. After these radiologic studies, she was advised close follow-up and repeat scan after 3 months.

On September 24, 2019, the patient had 4 to 5 episodes of tonic-clonic seizures, several bouts of projectile vomiting, weakness of all extremities, decreased verbal output and involuntary movement of both upper extremities. Pertinent physical examination showed a Glasgow Coma Scale (GCS) of 11 (E4M6V1), upper extremity myoclonus, 
and the manual muscle test (MMT) of all extremities are $4 / 5$.

Cranial CT-scan with contrast was done which shows a rimed, heterogeneously enhancing mass measuring 6.6 x $6.7 \times 8.0 \mathrm{~cm}$ in the region of the left fronto-temporoparietal lobe extending inferiorly to the left cranial fossa and is contiguous with the soft tissue lesion in the left pterygoid muscle. There are hyperdensities seen within the mass which may represent tumor bleed (Figure 1).

The patient underwent left temporal craniotomy with excision of the tumor. Histopathologic and cytogenetic studies of the cranial mass and slide review of the mandibular tumor were done which revealed rhabdomyosarcoma with spindle cell features of the brain occurring two years after the diagnosis of PNET/Ewing's sarcoma.

Three months after the diagnosis and initiation of treatment for rhabdomyosarcoma, the patient was admitted due to loss of consciousness. Plain and contrast cranial CT scan done showed an ill-defined, non-enhancing mass in the left tempo-parietal lobes measuring $7.0 \times 6.5 \times 8.0 \mathrm{~cm}$. There is noted soft tissue mass with calcifications and lytic destruction of the adjacent bone structures. Also noted are hydrocephalus, polysinusitis, right subfalcine herniation and brain edema of the left hemisphere (Figure 2). The consideration is tumor recurrence. The patient eventually succumbs to her illness and died.

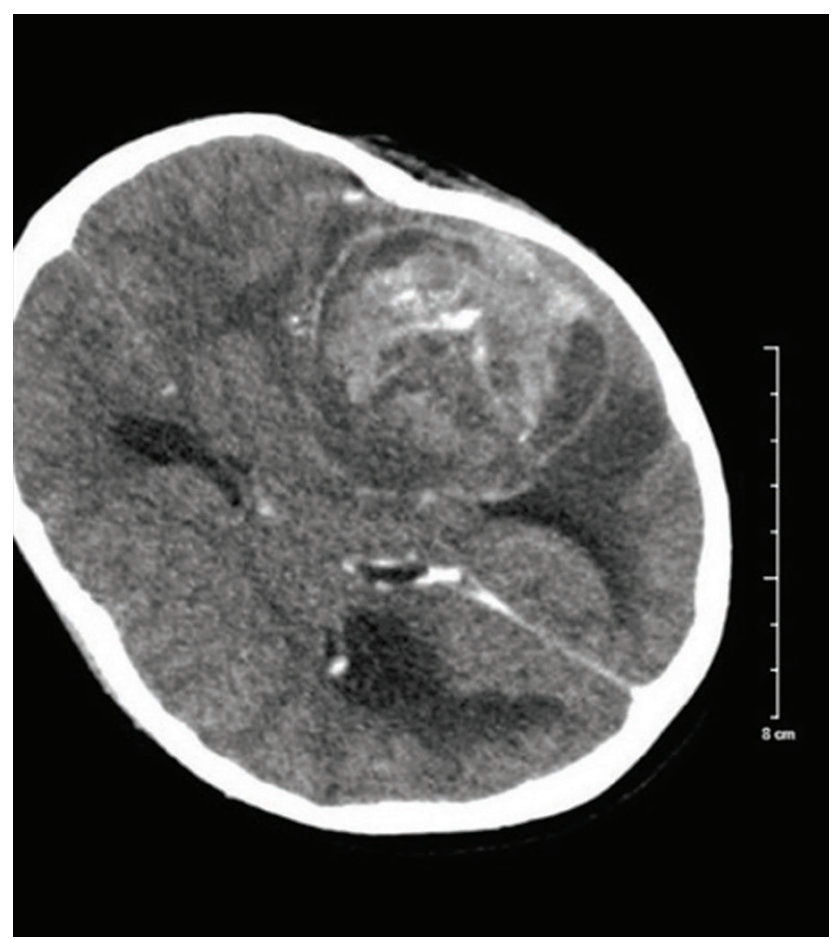

Figure 1. Cranial CT scan with contrast showed a 6.6 × $6.7 \times 8.0$ $\mathrm{cm}$ (AP T CC) mass, exhibiting rim and heterogeneous internal enhancement, in the region of the left fronto-temporo-parietal lobe. Hyperdensities are seen within the mass.

\section{PATHOLOGIC EXAMINATION AND CYTOGENETIC STUDIES}

\section{Oral cavity mass}

Slide review of hematoxylin and eosin (H\&E) stained smears show fibroconnective tissues containing salivary glands interspersed by malignant round-cell neoplasm with marked crushing artifact. The preserved, small round cells contain finely granular nuclei, prominent nucleoli and indistinct to clear cytoplasm. Immunohistochemical stains done showed strong, diffuse, membranous staining for cluster of differentiation (CD) 99 and negative for desmin and myogenin, supporting the diagnosis of a PNET/Ewing family of tumors (Figure 3). Cytogenetic studies for EWSR1 gene alteration to support PNET/ Ewing and FLI-1 to rule out rhabdomyosarcoma were further recommended.

The case was subsequently referred to a pediatric tertiary hospital. Immunohistochemistry performed at the said institution showed that the neoplastic cells are positive for CD99 and has diffuse nuclear positivity for myoblast determination protein 1 (MyoD1). Immunohistochemical staining for desmin, myogenin, CD34, S100 and SRY-Box transcription factor (SOX) 10 are all negative. Fluorescent in situ hybridization (FISH) targeting FOXO1 gene is negative. Reverse transcription polymerase chain reaction (RT-PCR) is positive for fusion transcript consistent

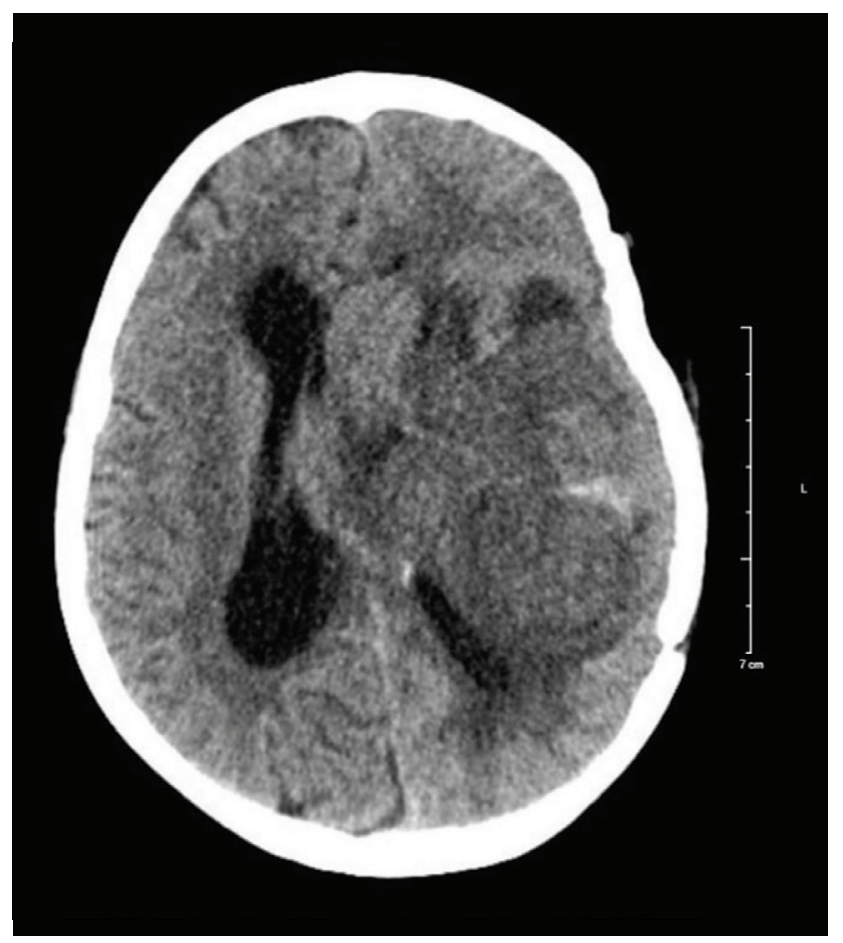

Figure 2. Plain and contrast CT scan showed a post craniotomy defect in the left fronto-parietal bones where in excision of the previous mass was noted. There is shift in the midline structures to the right with a finding of ill-defined non enhancing mass in the left fronto-parietal lobes measuring $7.0 \times 6.5 \times 8.0 \mathrm{~cm}$ replacing the malacic focus in the left parietal lobe. This lesion compresses the left lateral and third ventricles. There is effacement of the brain parenchyma in the left cerebral hemisphere and minimal perilesional edema above the lesion. 


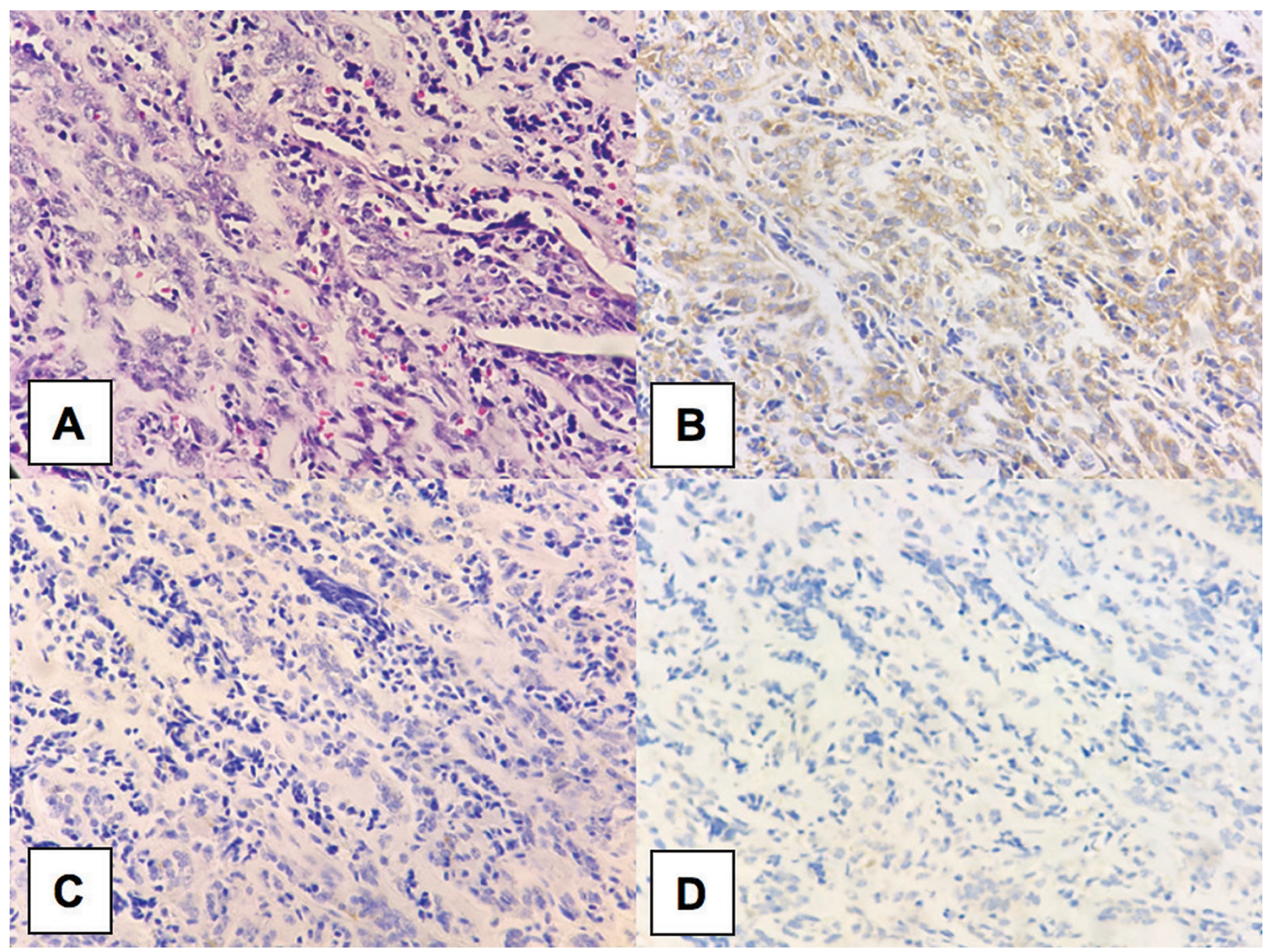

Figure 3. (A) H\&E shows small round blue neoplasm with marked crushing artifacts. The well preserved small, round cells have fine granular nuclei, some prominent nucleoli and indistinct to clear cytoplasm. (B) CD99 shows strong, diffuse, membranous immunohistochemical staining. (C and D) Immunohistochemical stains for desmin and myogenin are negative.

with Ewing's sarcoma/PNET family of tumors. These immunohistologic and cytogenetic findings are diagnosed as malignant round cell neoplasm with evidence of fusion transcript consistent with Ewing's sarcoma/PNET family of tumors by RT-PCR.

\section{Cerebral mass}

Two specimens are submitted for pathologic evaluation. One specimen is labeled as "Brain tumor 1" consisting of several cream, irregularly shaped, soft tissue fragments measuring $6.5 \times 6.5 \times 2.0 \mathrm{~cm}$ from which twenty-two representative sections are taken. The other specimen is labeled as "Brain tumor 2", consists of three cream, irregularly shaped, soft tissue fragments measuring $7.5 \mathrm{x}$ $6.7 \times 4.0 \mathrm{~cm}, 2.3 \times 2.0 \times 0.5 \mathrm{~cm}$, and $1.5 \times 1.3 \times 1.0 \mathrm{~cm}$ from which 24 representative sections are taken.

Microscopic examination shows sheets of medium to large spindle cells interspersed with patchy areas of necrosis. The cells contain increased nuclear to cytoplasmic ratio. The spindle cells have round to oval, vesicular nuclei with clumped chromatin patterns and slightly eosinophilic, scant cytoplasm. Admixed within the malignant cells are few round, spindle, and stellate cells containing ample eosinophilic cytoplasm with peripherally located nucleus and prominent nucleoli. There are 8-10 mitosis seen per high power field. Immunohistologic staining of the malignant cells show strong, nuclear reactivity for myogenin, strong, diffuse cytoplasmic reactivity for vimentin and rare cytoplasmic positivity for CD99 and BCL2 (Figure 4). The immunohistochemical stains are negative for CD34, CK and EMA. These findings are suggestive of rhabdomyosarcoma with spindle cell features.

The case was also referred to the previous referral institution, immunohistochemical stains done showed heterogeneous pattern of expression with desmin, myogenin and MyoD1. The tumor cells are negative for S100 and SOX10 with rare p53 expression. In line with the clinical history of PNET/Ewing sarcoma, reverse transcription polymerase chain reaction (RT-PCR) and fluorescence in situ hybridization (FISH) assay were performed in the brain lesion. These molecular tests turned out negative for EWSR1 gene alteration, suggesting that the two lesions are likely unrelated. This case was signed as morphologic and immunohistochemical findings consistent with rhabdomyosarcoma. 


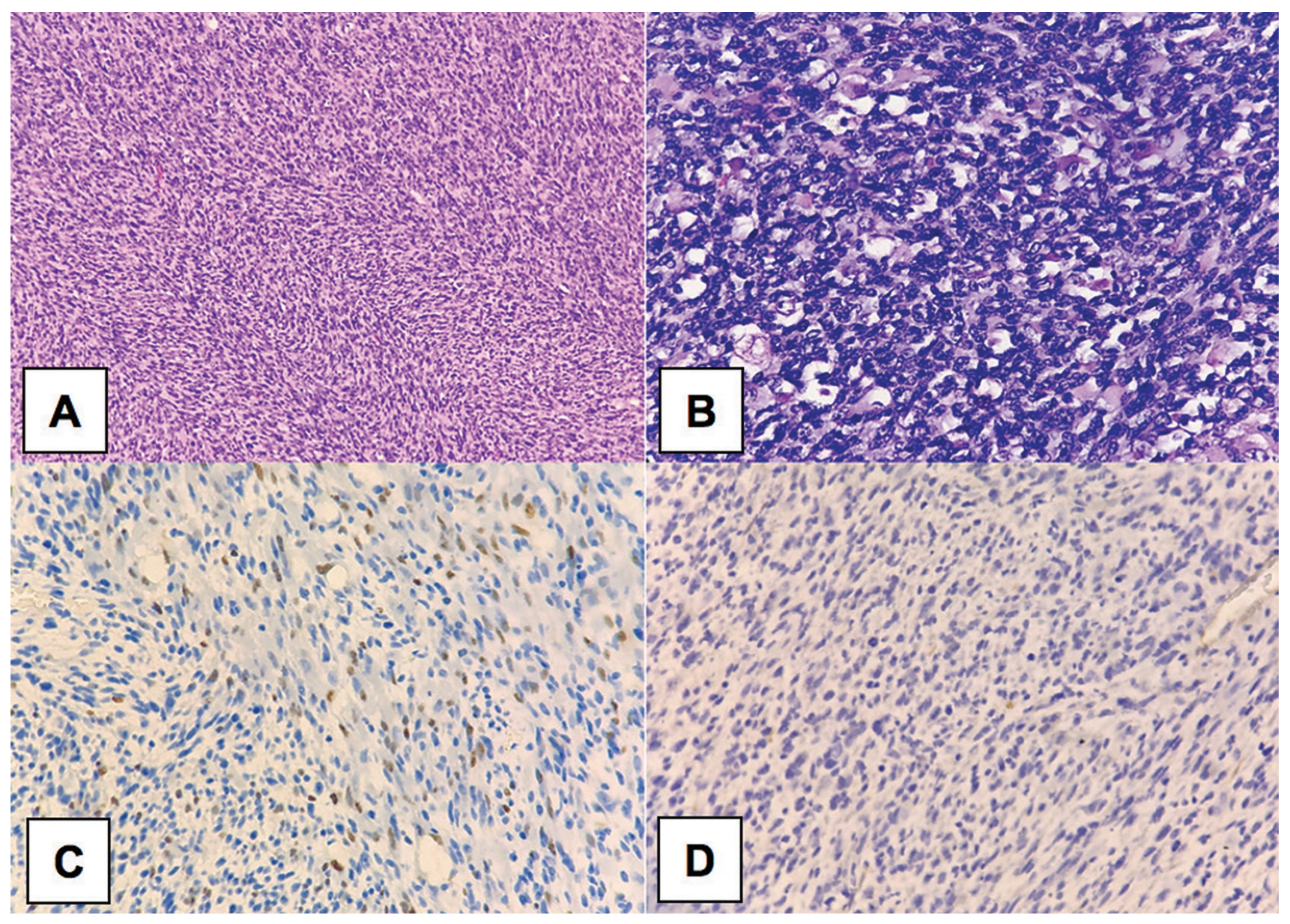

Figure 4. (A and B) Scanner and High Power Objective show sheets of medium to large spindle cells. These spindle cells have increased nuclear to cytoplasmic ration, slightly eosinophilic, scant cytoplasm, vesicular nuclei and clumped chromatin patterns. (C) Immunohistochemical staining for myogenin shows strong, and patchy nuclear reactivity. (D) Immunohistochemical staining for CD99 shows rare, cytoplasmic staining.

\section{DISCUSSION}

Multiple primary malignancies (MPM) are defined as two or more tumors arising from different organ systems. ${ }^{1}$ The entity was first published by Billroth in 1889, who noticed gastric carcinoma in a patient previously diagnosed with spinoepithelioma of the ear. ${ }^{2,6}$ Presently, the diagnosis of MPM follow the criteria established by Warren and Gates in $1932 ; 1$. Both the index and succeeding tumors are histologically different and confirmed as malignant, 2. The tumors occur in different organ system and separated by a normal mucosa, 3. The probability of metastasis from the index tumor is excluded. ${ }^{1,3}$ Furthermore, these malignancies are divided into two: synchronous - malignancies that are separated by less than six months and metachronous - if the succeeding malignancies occur more than six months from the diagnosis of the index tumor. ${ }^{1,2,4}$

The incidence of MPM ranges from $0.7 \%$ to $16 \%$. Moreover, synchronous and metachronous primary malignancy account for $25-36 \%$ and $67-75 \%$ respectively. ${ }^{1,2,5}$ Most of the index tumor are located in the head and neck, breast, gastrointestinal tract, gynecologic organs and genitourinary tract. On the other hand, secondary primary malignancies are more common in the gastrointestinal tract, lungs, gynecologic organs, breast and genitourinary tract. ${ }^{1,4}$

In spite of the abundant literatures addressing MPM, only few tackled multiple primary sarcomas. In a multicenter review involving 7351 patients previously diagnosed with soft tissue sarcomas in three tertiary orthopedic hospitals in Canada and United Kingdom, only six $(0.08 \%)$ had second primary sarcoma. ${ }^{7}$ The following factors are associated with new primary soft tissue sarcomas following previous resection of primary soft tissue sarcomas: 1. Genetic syndromes such as Li-Fraumeni syndrome, Hereditary Retinoblastoma, and Familial Adenomatous Polyposis, 2. History of radiation and chemotherapy, 3. Chronic inflammation, 4. Lymphedema, and 5. Exposure to chemical carcinogens. ${ }^{8}$ The 5 -year overall survival rate of multiple primary soft tissue sarcomas is $50 \%$, a steep decline from the $83.3 \%$ 5-year survival rate of the index cases of soft tissue sarcomas.

There are few reported sarcomas involving both PNET/ Ewing's sarcoma and rhabdomyosarcoma. Among these are five cases occurring simultaneously in the female genital tract. All of these cases are in the uterus with an average age of 39 years old and one reported pediatric case. Three of the cases are Fédération Internationale de 
Gynécologie et d'Obstétrique (FIGO) stage III with lymph node involvement, and two cases are localized in the uterus. It was further stated that the rhabdomyosarcoma component of the two localized neoplasms are of embryonal subtype, however the three other neoplasms were not histologically subclassified. Despite these neoplasms being immunohistomorphologically classified as PNET of the uterus, gene rearrangement for EWSR1 gene are not detected in all of the cases. ${ }^{9-13}$ De Alva et al., reported a case of a 13-year-old girl with a left arm mass who underwent fine needle aspiration biopsy. Cytologic evaluation showed strands of small cells with scant cytoplasm and hyperchromatic nuclei. Molecular study of this neoplasm showed simultaneous expression of the EWSR-FLI1 gene and PAX2-FKR gene, both are specific for Ewing's family of tumors (EFT) and alveolar rhabdomyosarcoma. ${ }^{14}$ Another series done in Canada, reported five cases of round cell neoplasms with immunohistomorphologic features compatible with rhabdomyosarcoma and simultaneous fusion of the EWSR1/FLI1 gene..$^{15}$

In contrast to the above mentioned cases, our case of an 11-year-old girl is unique since the diagnosis of rhabdomyosarcoma in the brain occurred two years after the diagnosis of PNET/Ewing's sarcoma in the oral cavity. Additionally, cytogenetic evaluation of both masses showed that EWSR1/FLI1 gene fusion seen in the oral cavity mass is not present in the brain mass, hence the conclusion that these tumors are likely unrelated. To the authors knowledge, there are no other case report on metachronous PNET/Ewing's sarcoma and rhabdomyosarcoma in the head and neck region and in the pediatric population.

The PNET/Ewing's sarcoma are grouped under EFT. These small, round cell neoplasms are neuroectodermal in origin. ${ }^{16}$ Microscopically, the cells are composed of small, round or oval cells with cytoplasm containing glycogen. ${ }^{17}$ Immunohistochemistry shows strong, and diffuse immunoreactivity for CD99 and Vimentin. ${ }^{16-17}$ An estimate of $85 \%$ of Ewing sarcomas has a somatic translocation involving $\mathrm{t}(11 ; 22)(\mathrm{q} 24 ; \mathrm{q} 12)$ that fuses EWSR1 to FLI $1 .{ }^{18}$ This translocation generates the EWSR1-FLI1 oncoprotein. ${ }^{18}$ Molecular diagnostic techniques such as RTPCR and FISH allow an accurate detection of the EWSR1FLI1 oncoprotein. The detection of this oncoprotein facilitates the differentiation from rhabdomyosarcoma, malignant lymphoma, desmoplastic small round cell tumor and other round cell neoplasms. ${ }^{16,17}$

A newly recognized group of malignancies known as "Ewing-like" neoplasms are emerging as diagnostic pitfalls. ${ }^{16-18}$ These group of neoplasm are morphologically similar to PNET/Ewing's sarcoma however lacks the molecular evidence supporting their inclusion in EFT. ${ }^{16-}$ ${ }^{18}$ Majority of the "Ewing-like" neoplasms have nuclear immunoreactivity for WT1 and $84 \%$ of these are positive for CD99 in a patchy staining pattern. ${ }^{17}$ The most common reported translocation in this group of tumors are the CIC-DUX4 fusion with few reported cases of BCORCCNB3 and CIC-FOXO4 fusion. ${ }^{17,18}$ It is important to recognize these tumors since the 5-year survival rate of "Ewing-like" neoplasms are $43 \%$, as compared to the $77 \%$ 5-year survival of Ewing's sarcoma. ${ }^{17}$
Rhabdomyosarcoma is a soft tissue neoplasm in which cells have propensity for myogenic differentiation. ${ }^{19}$ The World Health Organization (WHO) recognized four histologic variants of rhabdomyosarcoma. These variants are embryonal rhabdomyosarcoma, alveolar rhabdomyosarcoma, pleomorphic rhabdomyosarcoma and spindle cell/sclerosing rhabdomyosarcoma. ${ }^{18}$ The histologic examination of rhabdomyosarcoma varies per subtypes, but mostly these cells are heterogeneously shaped. $_{[19]}$ Some of these tumors contain undifferentiated cells, round to ovoid cells, "tadpole-like" cells, and spindle cells with eosinophilic cytoplasm and peripherally located nuclei known as rhabdomyoblasts. ${ }^{17-19}$ The rhabdomyoblasts may contain eosinophilic cytoplasm with thick and thin filaments appearing as striations. ${ }^{18}$

The most common subtype, Embryonal rhabdomyosarcoma shows tumor cells that are small and spindle in shape. ${ }^{17-18}$ A diagnostic feature of this subtype is the presence of highly cellular areas surrounding blood vessels alternating with variably cellular regions in a background of abundant myxoid material. ${ }^{17}$ Alveolar rhabdomyosarcoma contains small, round, or oval cells that are arranged in nests of connective tissue septa. ${ }^{17}$ Pleomorphic rhabdomyosarcoma are composed of large, atypical, and multinucleated cells that are difficult to differentiate from other soft tissue sarcomas. ${ }^{17}$ Diagnostic clues of pleomorphic rhabdomyosarcoma are the presence of rhabdomyoblasts and myognenin or MyoD1 immunoreactivity of malignant cells. ${ }^{17-18}$ Spindle cell rhabdomyosarcoma are predominantly composed of spindle cells with ovoid or elongated nuclei, vesicular chromatin, inconspicuous nucleoli and scant, pale eosinophilic cytoplasm. ${ }^{18}$ Presence of necrosis and high mitotic counts are common in this subtype. ${ }^{18}$ Few rhabdomyoblasts may be seen scattered within the spindle cells. ${ }^{18}$

Special examinations used to confirm the diagnosis of rhabdomyosarcoma are electron microscopy, immunohistochemical staining, and molecular studies. Electron microscopy allows the identification of sarcomere structures; however, these may not be present in poorly differentiated cells. ${ }^{17}$ Highly sensitive and specific immunohistochemical stains like myogenin and MyoD1 are commonly used to diagnose rhabdomyosarcoma. ${ }^{16} \mathrm{~A}$ specific indicator of muscle differentiation such as Desmin may be used, but this can stain both smooth and striated muscles. ${ }^{17}$ Cytogenetic testing for the diagnosis and prognosis of alveolar rhabdomyosarcoma to detect PAX3FOXO1 and PAX7-FOXOl genes using FISH and RTPCR may be used. ${ }^{16-19}$ In recent literature, approximately $75-78 \%$ of alveolar rhabdomyosarcomas are associated with the translocation $\mathrm{t}(2 ; 13)$ or $\mathrm{t}(1 ; 13)$, which result in the gene fusion PAX3-FOXO1 and PAX7-FOXO1. ${ }^{17-18}$

In a review article by Schiffman and Wright, subsequent malignant neoplasms after Ewing's sarcoma are subclassified into solid tumors and hematologic malignancies. The most common solid tumor is osteosarcoma (50-60\%) and the most common hematologic malignancy is acute myeloid leukemia or myelodysplastic syndrome $(60 \%)$. The article reviewed three censuses on the most common risk of childhood cancers that develop second malignancies; in both the British Census and the Surveillance, Epidemiology 
and End Result Program (SEER), Ewing's sarcoma is second, and in the multicenter cohort study done in France and United Kingdom, Ewing's sarcoma is ranked first. Based on the cumulative review, the onset of secondary malignancies due to Ewing's sarcoma 10 years after the diagnosis is at $5-6 \%$. In the report the main etiologies are the use of high dose (>60Gy) radiation therapy, DNA damage to hematopoietic stem cells due to activation of G-CSF by chemotherapy and the possibility of genetic abnormalities are being investigated. It was further stated that there is no predisposing genetic syndrome associated with Ewing's sarcoma, however translocation that encodes the EWS/FLI-1 protein can alter the RB and p53 pathway. ${ }^{20}$

\section{CONCLUSION}

Although there is no clear pathophysiology yet for MPM, the most common conjectures are genetic predisposition, iatrogenic and environmental exposure to carcinogens. Upon excluding metastasis, a low threshold for diagnosis of new primary malignancy should be suspected in previous cancer patients. An early diagnosis is essential for the management of multiple sarcomas since it has a remarkable decline in prognosis. Catching these malignancies at an early stage could be achieved by a harmonious coordination among the primary physician, hematologist and oncologist, radiologist and pathologist.

\section{ACKNOWLEDGMENT}

We would like to acknowledge the expertise of all the pathologists and clinicians that helped us in this case.

\section{ETHICAL CONSIDERATION}

Patient consent was obtained before submission of the manuscript.

\section{STATEMENT OF AUTHORSHIP}

All authors certified fulfillment of ICMJE authorship criteria.

\section{AUTHOR DISCLOSURE}

The authors declared no conflict of interest.

\section{FUNDING SOURCE}

None.

\section{REFERENCES}

1. Etiz D, Metcalfe E, Akcay M. Multiple primary malignant neoplasms: a 10-year experience at a single institution from Turkey. J Cancer Res Ther. 2017;13(1):16-20. PMID: 28508827. https://doi.org/ 10.4103/0973-1482.183219.

2. Guerriero A, Giovenali P, La Starza R, et al. Metachronous cardiac and cerebral sarcomas: case report with focus on molecular findings and review of the literature. Human Pathol. 2015;46(3):482-7. PMID: 25586016. https://doi.org/10.1016/j.humpath. 2014.10.028.
3. Warren S, Gates O. Multiple primary malignant tumors, a survey of the literature and statistical study. Am J Cancer 1932;16:1358-1414.

4. Zhang L, Feng L, Cong H, et al. Multiple primary malignant neoplasms: a case report and literature review. Oncol Lett. 2019; 18(4):4210-20. PMID: 31579423. PMCID: PMC6757307. https://doi.org/ 10.3892/ol.2019.10779.

5. Sharma D, Singh G, Kakkar N, Raj S. Second primary malignancy: a retrospective analysis report from a tertiary cancer center of North India. Indian J Cancer. 2016; 53(4), 595-9. PMID: 28485361. https://doi. org/10.4103/ijc.IJC_542_16.

6. Billroth T. Die allgemeine chirurgische Pathologie und Therapie in 50 Vorlesungen: Handbuch für Studierende und Ärztehttps. Winiwater A, ed. Germany: De Gruyter; 1883. https://doi. org/10.1515/9783111476230. https://www.degruyter. com/view/title/57990.

7. Lex JR, Aoude A, Stevenson JD, et al. Multiple soft tissue sarcomas in a single patient: an international multicentre review. Sarcoma. 2018:Article ID 5392785. https://doi.org/10.1155/2018/5392785.

8. Witt RG, Baldini EH, Raut CP. Screening populations at high risk for soft tissue sarcoma and surveillance following soft tissue sarcoma resection. J Surg Oncol. 2019;120(5):882-90. PMID: 31432526. https://doi. org/10.1002/jso.25676.

9. Cate F, Bridge JA, Crispens MA, et al. Composite uterine neoplasm with embryonal rhabdomyosarcoma and primitive neuroectodermal tumor components: rhabdomyosarcoma with divergent differentiation, variant of primitive neuroectodermal tumor, or unique entity? Hum Pathol. 2013;44(4):656-63. PMID: 23266445. https://doi.org/10.1016/j.humpath. 2012.09.008.

10. Chang L, Enriquez M, Lerman N, WilsonSmith R. High grade sarcoma, with predominant neuroectodermal and minor embryonal rhabdomyosarcomatous tumor of the uterus: a case report. Gynecol Oncol Rep. 2019;28:128-32. PMID: 31032392. PMCID: PMC6479011. https://doi.org/10.1016/j.gore. 2019.04.001.

11. Stolnicu S, Goyenaga P, Hincu M, et al. Embryonal (botryoides) rhabdomyosarcoma of the uterus harboring a primitive neuroectodermal tumor component. Int J Gynecol Pathol. 2012;31(4): 387-9. PMID: 22653355. https://doi.org/10.1097/ PGP.0b013e31823ff3e6

12. Dundr P, Fischerová D, Povýšil C, et al. Uterine tumors with neuroectodermal differentiation. a report of 4 cases. Pathol Oncol Res. 2010;16(4): 601-8. PMID: 20204716. https://doi.org/10.1007/ s12253-010-9249-7.

13. Euscher ED, Deavers MT, Lopez-Terrada D, et al. Uterine tumors with neuroectodermal differentiation: a series of 17 cases and review of the literature. Am J Surg Pathol. 2008;32(2):219-28. PMID: 18223324. https://doi.org/10.1097/PAS.0b013e318093e421.

14. De Alava E, Lozano M, Sola I, et al. Molecular features in a biphenotypic small cell sarcoma with neuroectodermal and muscle differentiation. Human Pathol. 1998;29(2):181-4. PMID: 9490279. https:// doi.org/10.1016/s0046-8177(98)90230-1. 
15. Sorensen PH, Shimada H, Liu XF, Thomas G, Triche TJ. Biphenotypic sarcomas with myogenic and neural differentiation express the Ewing's sarcoma EWS/FLI1 fusion gene. Cancer Res. 1955;55(6):138592. PMID: 7882340.

16. Dome JS, Rodriguez-Galindo C, Spunt SL, Santana VM. Pediatric solid tumors, In Abeloff MD, ed. Abeloff's Clinical Oncology, 4th ed. Philadelphia, Pennsylvania: Elsevier Health Sciences; 2008.

17. Ackerman LV, Goldblum JR, Lamps LW, McKenney JK, Myers JL, Rosai J. Rosai and Ackerman's Surgical Pathology, 11th ed. Philadelphia, Pennsylvania: Elsevier; 2018.
18. Fletcher CDM, Bridge JA, Hogendroorn PCW, Mertens F, eds. WHO classification of tumours of soft tissue and bone. Lyon: International Agency for Research on Cancer; 2013.

19. Skapek SX, Ferrari A, Gupta AA, et al. (2019). Rhabdomyosarcoma. Nature Rev Dis Primers. 2019;5(1):1. PMID: 30617281. PMCID: PMC7456566. https://doi.org/10.1038/s41572-0180051-2.

20. Schiffman JD, Wright J. Ewing's sarcoma and second malignancies. Sarcoma, 2011: Article ID 736841. https://doi.org/10.1155/2011/736841.

Disclaimer: This journal is OPEN ACCESS, providing immediate access to its content on the principle that making research freely available to the public supports a greater global exchange of knowledge. As a requirement for submission to the PJP, all authors have accomplished an AUTHOR FORM, which declares that the ICMJE criteria for authorship have been met by each author listed, that the article represents original material, has not been published, accepted for publication in other journals, or concurrently submitted to other journals, and that all funding and conflicts of interest have been declared. Consent forms have been secured for the publication of information about patients or cases; otherwise, authors have declared that all means have been exhausted for securing consent.

\section{Publish in the new PJP. Visit our website: http://philippinejournalofpathology.org}

